the elements of its first column vanish identically. The proof applied to $D$ is therefore applicable to this determinant and the theorem is proved in general.

HaRVARD UNIVERSITY, June 1, 1916.

\title{
NOTE ON THE LINEAR DEPENDENCE OF ANALYTIC FUNCTIONS.
}

BY DR. G. A. PFEIFFER.

(Read before the American Mathematical Society, September 5, 1916.)

The theorem proved in the preceding note, that a necessary and sufficient condition that $p$ analytic functions, $f_{1}(t), f_{2}(t)$, $\cdots, f_{p}(t)$, be linearly dependent is that the determinant whose $i$ th row is

$$
\begin{gathered}
f_{i}^{\left(\lambda_{1}\right)}\left(t_{1}\right), f_{i}^{\left(\lambda_{1}-1\right)}\left(t_{1}\right), \cdots, f_{i}\left(t_{1}\right), f_{i}^{\left(\lambda_{2}\right)}\left(t_{2}\right), f_{i}^{\left(\lambda_{2}-1\right)}\left(t_{2}\right), \cdots, f_{i}\left(t_{2}\right), \\
\cdots, \cdots, f_{i}^{\left(\lambda_{\mu}\right)}\left(t_{\mu}\right), f_{i}^{\left(\lambda_{\mu}-1\right)}\left(t_{\mu}\right), \cdots, f_{i}\left(t_{\mu}\right) \\
\left(i=1,2, \cdots, p ; p=\mu+\sum_{i=1}^{\mu} \lambda_{i}\right)
\end{gathered}
$$

vanish identically in $t_{1}, t_{2}, \cdots, t_{p}$, can be readily proved if we assume the fundamental theorem that the identical vanishing of the wronskian of $p$ analytic functions implies their linear dependence.

By rearranging the columns of the determinant of the theorem we obtain the determinant $\Delta$ whose $i$ th row is

$$
\begin{array}{r}
f_{i}\left(t_{1}\right), f_{i}{ }^{\prime}\left(t_{1}\right), \cdots, f_{i}{ }^{\left(\lambda_{1}\right)}\left(t_{1}\right), f_{i}\left(t_{2}\right), f_{i}{ }^{\prime}\left(t_{2}\right), \cdots, f_{i}^{\left(\lambda_{2}\right)}\left(t_{2}\right), \\
\cdots, \cdots, f_{i}\left(t_{\mu}\right), f_{i}{ }^{\prime}\left(t_{\mu}\right), \cdots, f_{i}{ }^{\left(\lambda_{\mu}\right)}\left(t_{\mu}\right) .
\end{array}
$$

Without losing any generality we shall assume that

$$
\lambda_{1} \geqq \lambda_{2} \geqq \cdots \geqq \lambda_{\mu} .
$$

Now the derivative of order $n q$ of the $q$-rowed determinant whose $i$ th row $(i=1,2, \cdots, q)$ is

$$
f_{i}(t), f_{i}^{\prime}(t), \cdots, f_{i}^{(q-1)}(t)
$$

is equal to a positive integer times the $q$-rowed determinant whose $i$ th row is 


$$
f_{i}^{(n)}(t), f_{i}^{(n+1)}(t), \cdots, f_{i}^{(n+q-1)}(t)
$$

plus a sum of $q$-rowed determinants each of which has at least one column consisting of the derivatives of $f_{1}(t), f_{2}(t)$, $\cdots, f_{q}(t)$ of an order less than $n$.

Using this fact we find upon differentiating the determinant $\Delta\left(\lambda_{1}+1\right)\left(\lambda_{2}+1\right)$ times with respect to $t_{2}$ and putting $t_{2}=t_{1}$ that the result is equal to a positive integer multiplied by the $p$-rowed determinant whose $i$ th row is

$$
\begin{aligned}
f_{i}\left(t_{1}\right), f_{i}{ }^{\prime}\left(t_{1}\right), \cdots, f_{i}^{\left(\lambda_{1}\right)}\left(t_{1}\right), f_{i}{ }^{\left(\lambda_{1}+1\right)}\left(t_{1}\right), \cdots, f_{i}{ }^{\left(\lambda_{1}+\lambda_{2}+1\right)}\left(t_{1}\right), \\
f_{i}\left(t_{3}\right), \cdots, f_{i}^{\left(\lambda_{3}\right)}\left(t_{3}\right), \cdots, \cdots, f_{i}\left(t_{\mu}\right), \cdots, f_{i}{ }^{\left(\lambda_{\mu}\right)}\left(t_{\mu}\right) .
\end{aligned}
$$

The other determinants which result from the differentiation drop out when $t_{2}$ is put equal to $t_{1}$, since each of them then has at least two columns identical. Repeating this process for the other variables in turn, we finally have the $p$-rowed determinant whose $i$ th row is

$$
f_{i}\left(t_{1}\right), f_{i}{ }^{\prime}\left(t_{1}\right), \cdots, f_{i}{ }^{(p-1)}\left(t_{1}\right)
$$

(or the wronskian of the $p$ given functions) vanishing identically if the determinant of the theorem does. The necessity of the condition of the theorem follows immediately as in the proof concerning the wronskian.

HARVARD UNIVERSITY,

July, 1916.

\title{
ON THE LINEAR DEPENDENCE OF FUNCTIONS OF ONE VARIABLE.
}

\author{
BY DR. G. M. GREEN.
}

(Read before the American Mathematical Society, September 5, 1916.)

As is well known, the identical vanishing of the wronskian of $p$ functions of a single variable is a sufficient condition for their linear dependence if the functions are analytic; if, however, they are not analytic the vanishing of the wronskian is not sufficient. The same situation arises in connection with the theorem proved by Mr. Morse and by Dr. Pfeiffer in the present number of the BuLletin. The former makes explicit use of the analytic character of the functions involved, whereas the theorem proved by the latter may be neatly stated only for analytic functions, if it is to afford a criterion for linear dependence. 